\title{
Anisotropic Electric Conductivity and Battery Performance in $C$-axis Oriented Lanthanum Silicate Oxyapatite Prepared by Slip Casting in a Strong Magnetic Field $^{* 1}$
}

\author{
Tohru S. Suzuki ${ }^{1, * 2}$, Satoshi Takahashi ${ }^{2}$, Tetsuo Uchikoshi ${ }^{1}$, Takamasa Ishigaki ${ }^{2}$ and Kiyoshi Kobayashi ${ }^{1}$ \\ ${ }^{1}$ Research Center for Functional Materials, National Institute for Materials Science, Tsukuba 305-0047, Japan \\ ${ }^{2}$ Department of Chemical Science and Technology, Hosei University, Tokyo 184-8584, Japan
}

Lanthanum silicate oxyapatite (LSO) has superior properties as a solid electrolyte for solid oxide fuel cell (SOFC) applications. This is because LSO has a higher oxide-ion conductivity compared to yttria-stabilized zirconia at temperatures below $600^{\circ} \mathrm{C}$. Textured LSO bulk ceramics were fabricated based on a magnetic field-assisted colloidal processing technique. The $c$-axis of the LSO was aligned parallel to the applied magnetic field during the consolidation by slip casting. The anisotropic electric conductivity of the textured bulk ceramics was evaluated by the impedance spectroscopy method. It was confirmed that very high conductivity was obtained along the $c$-axis. Higher fuel cell performance was demonstrated in the textured LSO compared to the random LSO. [doi:10.2320/matertrans.Y-M2019832]

(Received February 14, 2019; Accepted May 28, 2019; Published July 22, 2019)

Keywords: orientation, magnetic field, sintering, electric conductivity, electric power generation

\section{Introduction}

Lanthanoid silicate oxyapatites $\left(\mathrm{RE}_{\mathrm{x}} \mathrm{Si}_{6} \mathrm{O}_{12+1.5 \mathrm{x}}, \mathrm{RE}=\right.$ $\mathrm{La}, \mathrm{Nd}, \mathrm{Sm}, \mathrm{Gd}$, or Dy, $\mathrm{x}=8-11$ ) are novel oxide-ion conductors, and were discovered by Nakayama et al. in 1995. ${ }^{1,2)}$ Apatite-type lanthanum silicate $(\mathrm{RE}=\mathrm{La}$, hereafter referred to as LSO) has the highest oxide-ion conductivity among Lanthanoid silicates, and possesses a high oxide-ion conductivity and a low-activation energy of oxide-ion conduction. It has been reported that the oxide-ion conductivity in a medium temperature range is higher than that of the typical oxide-ion conductor of yttria-stabilized zirconia (YSZ). ${ }^{3)}$ Furthermore, it does not contain easilyreducible elements and it is chemically stable in a wide range of oxygen partial pressure. It is therefore attracting attention as an electrolyte of a next generation type solid oxide fuel cell operating in a medium and low temperature range. ${ }^{4)}$ It has been reported that the single crystals of $\mathrm{RE}_{9.33}\left(\mathrm{SiO}_{4}\right)_{6} \mathrm{O}_{2}$ $(\mathrm{RE}=\mathrm{Pr}, \mathrm{Nd}, \mathrm{Sm})$ fabricated from 1999 to 2004 exhibit anisotropic ionic conductivity. ${ }^{5-10)}$ This is a very interesting feature, and this anisotropy is not encountered in other oxideion conductors, such as yttria-stabilized zirconia, scandiastabilized zirconia with fluorite crystal structures, and lanthanum gallate systems with perovskite crystal structures. The crystal structure of LSO, shown in Fig. 1, is hexagonal apatite structure. Based on the single-crystal X-ray diffraction and powder neutron diffraction, it has been reported that the probability density distribution of the oxide ion at the O4 site is highly anisotropic and elongated along the $c$-axis direction. ${ }^{11-13)}$ Furthermore, it has been reported that in a single LSO crystal, the oxide-ionic conductivity in the $c$-axis direction is higher than those along the other axes. ${ }^{14)}$ Therefore, it is expected that the ionic conductivity can be improved by the alignment of the $c$-axis, even in polycrystalline LSO.

\footnotetext{
${ }^{* 1}$ This Paper was Originally Published in Japanese in J. Jpn. Soc. Powder Powder Metallurgy 65 (2018) 121-126.

${ }^{* 2}$ Corresponding author, E-mail: suzuki.tohru@nims.go.jp
}

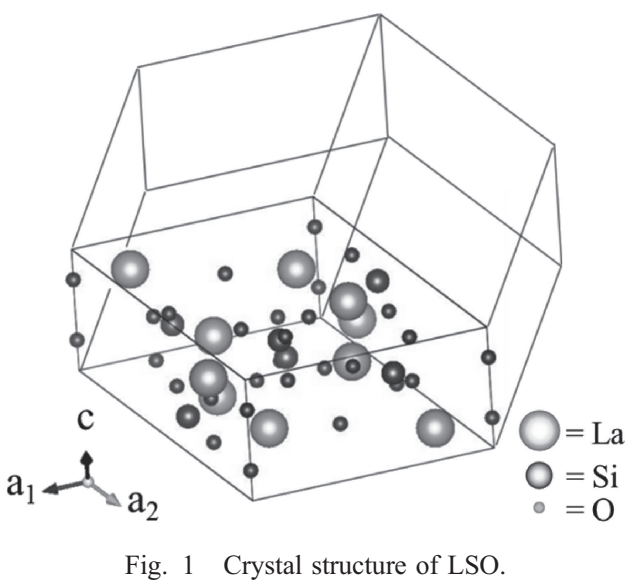

Fukuda et al. demonstrated that the ionic conductivity of LSO can be improved by developing a columnar microstructure with $c$-axis orientation fabricated by the isothermal heating of the sandwich-type $\mathrm{La}_{2} \mathrm{SiO}_{5} / \mathrm{La}_{2} \mathrm{Si}_{2} \mathrm{O}_{7}$ pseudodiffusion couple. ${ }^{15)}$ Regarding to the different method, it has been reported that the LSO with $c$-axis orientation could be fabricated from $\mathrm{La}_{10}\left(\mathrm{SiO}_{4}\right)_{6} \mathrm{O}_{3}$ powder containing $\mathrm{La}_{2} \mathrm{SiO}_{5}$ phase as an impurity in the presence of a magnetic field with a strength of $10 \mathrm{~T}$ followed by sintering. ${ }^{16)}$ However, the Lotgering factor indicating orientation degree index was as low as 0.452 . Consequently, a higher degree of orientation should be achieved to enhance the ionic conductivity. The initial powder size and shape are the two factors that determine orientation in the presence of a magnetic field. We therefore expect that highly oriented LSO can be fabricated by optimizing the size and shape of powder and powder dispersion in the slurry.

In the present study, the slurry condition was controlled to enhance the orientation of the LSO with respect to the direction of the magnetic field. Accordingly, it was demonstrated that the textured LSO exhibits anisotropic fuel cell performance. 


\section{Experimental Procedure}

$\mathrm{La}_{2} \mathrm{O}_{3}$ powder, which was heated for eliminating the hydroxide and carbonate before weighing, and $\mathrm{SiO}_{2}$ powder were dispersed in distilled water and mixed with ball milling. ${ }^{17)}$ The mixture was then dried at $130^{\circ} \mathrm{C}$. Subsequently, this mixture was calcined at $400^{\circ} \mathrm{C}$ and $600^{\circ} \mathrm{C}$ for $10 \mathrm{~h}$. This calcined powder was pulverized with ball milling, and the $\mathrm{La}_{9.33}\left(\mathrm{SiO}_{4}\right)_{6} \mathrm{O}_{2}$ phase was synthesized by a solidstate reaction at $1400^{\circ} \mathrm{C}$ for $12 \mathrm{~h}$. The synthesized powder was then pulverized to a submicron size with ball milling, and the submicron-sized powder was used as the initial powder.

This initial powder was dispersed in distilled water with an appropriate amount of dispersant (ammonium polycarboxylate, A6114, Toagosei Co., Ltd.) with ball milling using a polyethylene container and zirconia balls with a diameter of $5 \mathrm{~mm}$. For comparison, the slurries were consolidated by slip casting in the absence or presence of a magnetic field of $12 \mathrm{~T}$. The green compacts were dried in air and then further densified using cold isostatic pressing at $392 \mathrm{MPa}$ for $10 \mathrm{~min}$ without disturbing the particle orientation. Final sintering was conducted at $1600^{\circ} \mathrm{C}$ for $10 \mathrm{~h}$ in air.

To observe the microstructure and analyze the crystallographic orientation by electron backscattering diffraction (EBSD), the LSO surface perpendicular to the magnetic field was polished to form a mirror surface. Orientation imaging microscopy ${ }^{\mathrm{TM}}$ (TexSEM Laboratories, Inc.) was used for the acquisition and analyses of the EBSD data. The total electrical-conductivities of the sintered $\mathrm{La}_{9.33}\left(\mathrm{SiO}_{4}\right)_{6} \mathrm{O}_{2}$ with random and $c$-axis orientations were measured using the alternating current two-probe impedance method in air using a potentiogalvanostat (Versastat4, Princeton Applied Research, USA). Platinum paste (TR-7907, Tanaka Kikinzoku Kogyo. Co. Ltd., Japan) was painted on the top and bottom surfaces of the sintered $\mathrm{La}_{9.33}\left(\mathrm{SiO}_{4}\right)_{6} \mathrm{O}_{2}$, and then heated at $1000^{\circ} \mathrm{C}$ for $30 \mathrm{~min}$ to make electrodes on both the surfaces. The frequency ranges were varied from $1 \mathrm{MHz}$ to $1 \mathrm{mHz}$ at fixed temperatures and the fixed temperature range was varied from 350 to $1000^{\circ} \mathrm{C}$. It was difficult to separately distinguish grain boundary resistance from the collected impedance spectra and therefore, the total conductivity was calculated by sum of the bulk and grain boundary resistances. Consequently, the entire resistance was determined to evaluate the total electric conductivity. Furthermore, the activation energy was calculated based on the Arrhenius plot.

Coin-type single-fuel cell performance was quantified based on $I-V$ measurements. The sintered $\mathrm{La}_{9.33}\left(\mathrm{SiO}_{4}\right)_{6} \mathrm{O}_{2}$ ceramics with random or $c$-axis orientations fabricated by the above-method constituted the solid electrolytes. The prepared pellet samples had a diameter of $25 \mathrm{~mm}$ and were ground to a thickness of $\sim 0.9 \mathrm{~mm}$. To eliminate the properties based on the electrolyte materials and the interface between the electrode and the sintered $\mathrm{La}_{9.33}\left(\mathrm{SiO}_{4}\right)_{6} \mathrm{O}_{2}$ electrolyte, $\mathrm{Pt}$ paste (TR-7907, Tanaka Kikinzoku Kogyo. Co. Ltd., Japan) (which were often chosen as the test electrodes) was used as both the fuel and air electrodes. The Pt paste was painted on the top and bottom surfaces of the sintered $\mathrm{La}_{9.33}\left(\mathrm{SiO}_{4}\right)_{6} \mathrm{O}_{2}$ after polishing with the use of $15 \mu \mathrm{m}$ polishing media, and was heated at $1000^{\circ} \mathrm{C}$ for $30 \mathrm{~min}$. Air and hydrogen were flowed at $100 \mathrm{~cm}^{3} / \mathrm{min}$ on the cathode and the anode during the $I-V$ measurements, respectively. To measure the cell temperature, the R-type thermocouple was placed near the anode surface. $I-V$ measurement was conducted in the temperature range of $400-1000^{\circ} \mathrm{C}$ at $100^{\circ} \mathrm{C}$ intervals. The potentio/galvanostat (VSP, BioLogic Science Instruments, France) was used for the evaluation of the cell operation performance.

\section{Results and Discussion}

Figure 2 shows the morphologies of the synthesized powder which had an average particle diameter of $0.9 \mu \mathrm{m}$ after pulverizing. Figure 3 illustrates the XRD profiles of the pulverized powder and the sintered specimens prepared in the absence of a magnetic field. It was verified that though an amorphous-like phase and the impurities were observed at low angles, all the other reflections were identified by the $\mathrm{La}_{9.33}\left(\mathrm{SiO}_{4}\right)_{6} \mathrm{O}_{2}$ phase in the pulverized powder. The same impurity phase remained in the sintered sample with a random orientation. Figure 4 shows the zeta potential of the pulverized powder in the water-based slurry prepared with dispersant. It is clear that the $\mathrm{pH}$ value became equal to 9.5 just after the preparation of the slurry which contained the pulverized powder and the dispersant, while the zeta potential

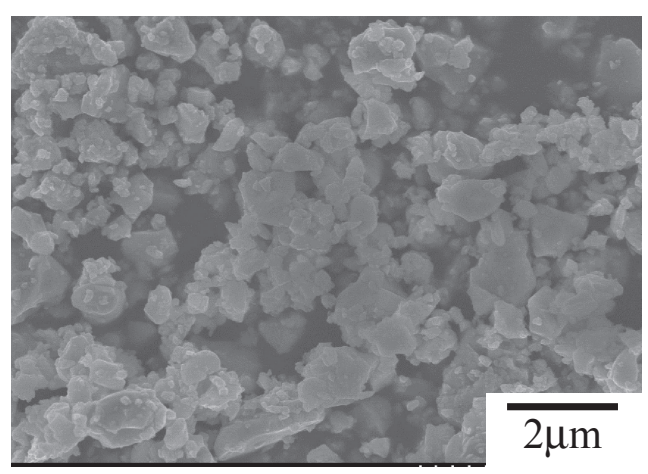

Fig. 2 Photo of milled LSO powder.

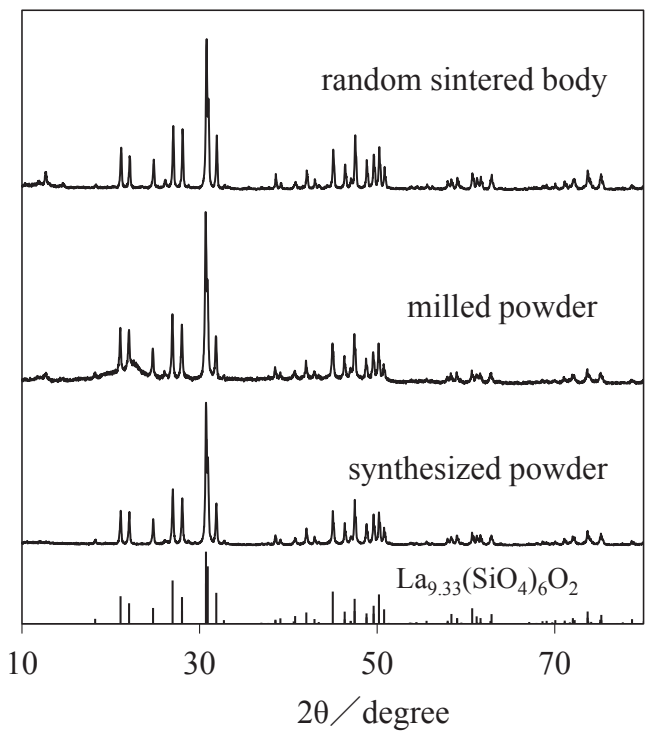

Fig. 3 XRD profiles of the synthesized, milled LSO powder and random sintered specimen. 


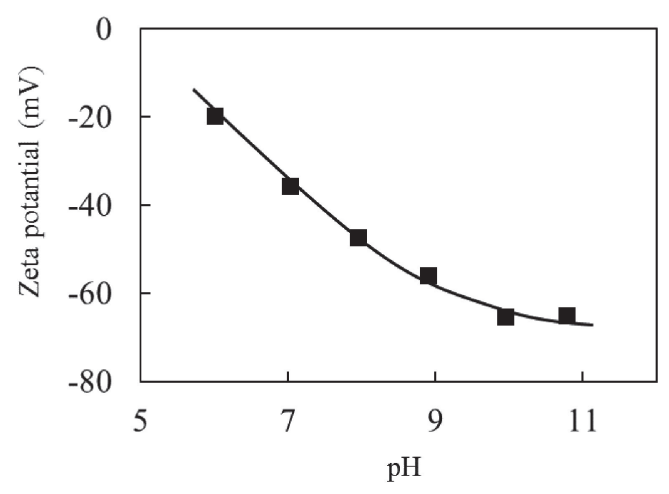

Fig. 4 Zeta potential of the milled powder in water based slurry.

was of sufficient magnitude in negative charge to achieve particle dispersion at the $\mathrm{pH}$ higher than 9.5. Figure 5(a) and (b) illustrate the image quality maps of the EBSD measurements on the plane perpendicular to the direction of the magnetic field, and on the vertical plane to the slip casting direction in the sintered specimens in the presence and absence of the magnetic field, respectively. These maps are plots of the peak intensity indicated by the band in the Hough space in the EBSD analyses, and depend on the crystallinity and the surface conditions. Consequently, the gray shading does not represent information on composition, but merely shows that the grain shape and size are similar to the microstructural observations of the acquired SEM images. This is because the crystallinity decreases, and the gray shading is changed to dark gray at the grain boundary. The average grain sizes are approximately $6.5 \mu \mathrm{m}$, and they are approximately the same for specimens with random and $c$-axis orientations.

The pole figure constructed based on the EBSD analyses is shown in Fig. 6. In the sintered sample prepared in the absence of the magnetic field, the intensity distribution is almost uniform on any crystalline plane, such as the $\{0001\}$ plane, exhibiting a random orientation. By comparison, in the sample prepared by slip casting in the presence of the magnetic field, the $\{0001\}$ pole figure shows a very intense
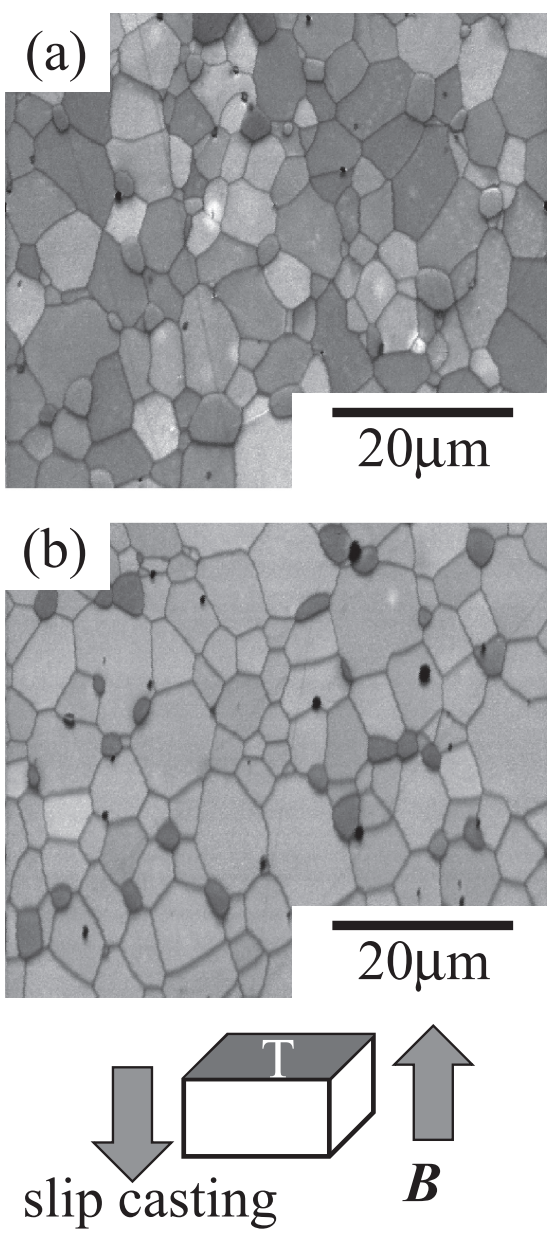

Fig. 5 Microstructures of LSO prepared by slip casting (a) outside or (b) in a magnetic field followed by sintering at $1600^{\circ} \mathrm{C}$.

spot at the center, which indicates that the $c$-axis was aligned along the direction of the magnetic field with the highintensity ring being at the periphery in the $\{10 \overline{1} 0\}$ pole figure. This indicates that the $a$-axis orientation is radially symmetric within the plane of the T-surface perpendicular to the

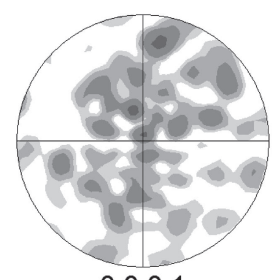

0001

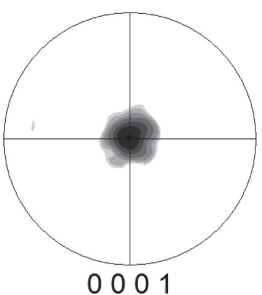

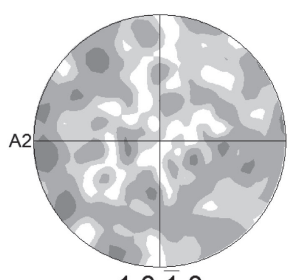

$10 \overline{10}$

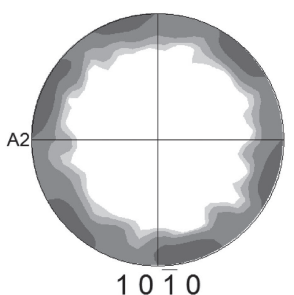

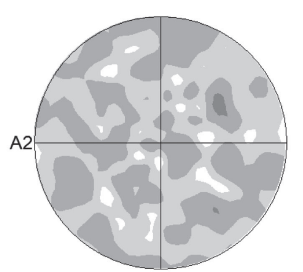

1121

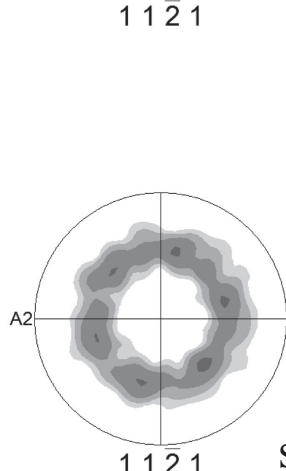

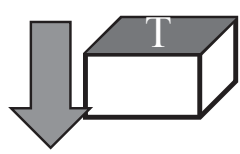

slip casting

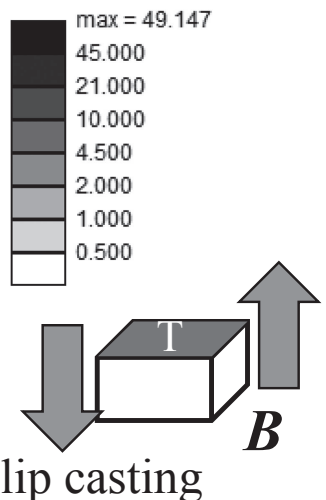

Fig. 6 Pole figure on T-plane in the LSO prepared by slip casting outside or in a magnetic field followed by sintering at $1600^{\circ} \mathrm{C}$. 


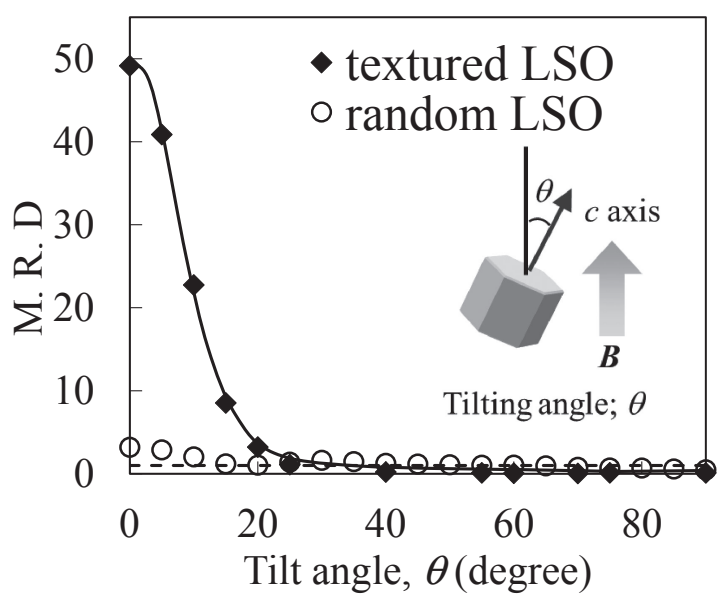

Fig. 7 Distribution of tilt angle between the $c$-axis and vertical direction in the LSO prepared by slip casting outside or in a magnetic field followed by sintering at $1600^{\circ} \mathrm{C}$.

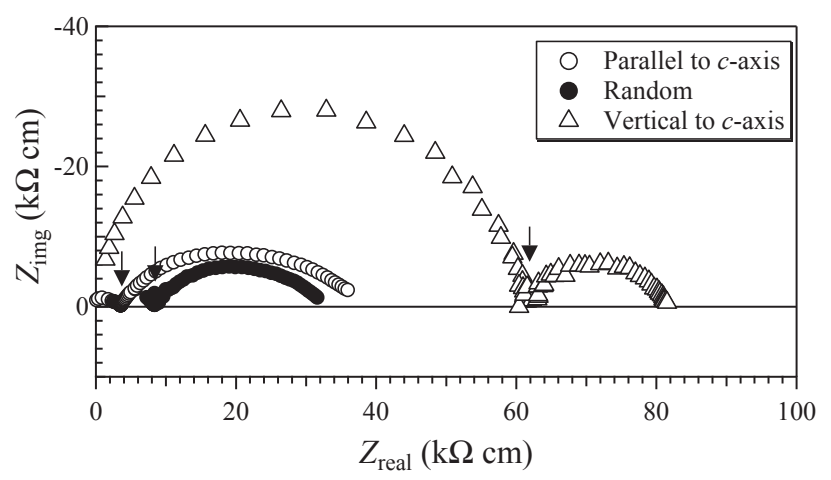

Fig. 8 Nyquist plots of the LSO prepared by slip casting outside or in a magnetic field followed by sintering at $1600^{\circ} \mathrm{C}$. Measurement temperature is $600^{\circ} \mathrm{C}$.

magnetic field. Therefore, it is clear that the $c$-axis orientation can be controlled by the magnetic field.

The multiples of a random distribution (MRD) calculated based on the EBSD analyses were plotted as a function of the tilt angle between the $c$-axis and the vertical direction parallel to the magnetic field as shown in Fig. 7. In the sample prepared in the absence of the magnetic field, MRD was independent of the tilting angle. By contrast, the profile of the sample prepared in the presence of the magnetic field indicates that $77 \%$ of the grains were aligned with the tilt angle less than $20^{\circ}$ while $45 \%$ of grains were aligned with the tilt angle less than $10^{\circ}$. Furthermore, only $2.2 \%$ of the grains were aligned with angles larger than $45^{\circ}$.

Figure 8 shows the impedance plots for the direction parallel and perpendicular to the $c$-axis, and for random orientations at $600^{\circ} \mathrm{C}$. Figure 9 illustrates Arrhenius plots of the total conductivity calculated from the resistance values indicated by the black arrows in Fig. 8. The conductivity parallel to the $c$-axis was the highest, while that perpendicular to the $c$-axis was the lowest, as also reported for single crystals. The conductivity of the sample with random orientation was between the two values obtained on the direction parallel and perpendicular to the $c$-axis. It was verified that the $c$-axis orientation controlled by the magnetic field alignment, was very effective to improve the

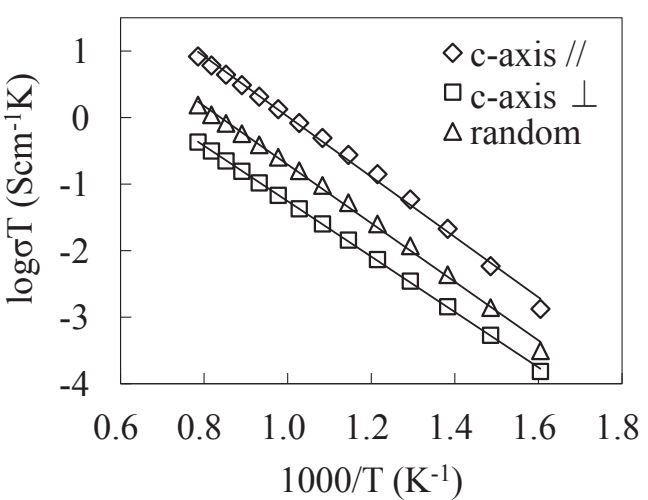

Fig. 9 Electric conductivity of the LSO prepared by slip casting outside or in a magnetic field followed by sintering at $1600^{\circ} \mathrm{C}$.

conductivity of the textured polycrystalline $\mathrm{La}_{9.33}\left(\mathrm{SiO}_{4}\right)_{6} \mathrm{O}_{2}$. The activation energies of the oxide-ion conduction parallel and perpendicular to the $c$-axis, and in samples with random orientations, were $0.90 \mathrm{eV}, 0.83 \mathrm{eV}$ and $0.82 \mathrm{eV}$, respectively. Although it was not clear that the activation energy was anisotropic, depending on the $c$-axis direction, it was clarified that the conductivities parallel and perpendicular to the $c$-axis were anisotropic. The ionic conductivity $\left(\sigma_{i}\right)$ can be expressed by the following equation.

$$
\sigma_{i}=\frac{A \gamma q_{i}^{2} c_{i} a^{2}}{k T} \cdot \exp \left(-\frac{\Delta H_{m}}{k T}\right)
$$

where $A$ is area of cross-section, $\gamma$ is the frequency factor depending on the crystal direction, $q_{i}$ is the valence of ions, $c_{i}$ is the concentration of conductive ion, $a$ is the jump distance, $k$ is the Boltzmann constant, $T$ is the absolute temperature, and $\Delta H_{m}$ is the activation energy for oxide-ion conduction. Even if $\Delta H_{m}$ is independent of the crystal direction, the anisotropic conductivity depends on the crystal direction, as observed. It is considered that the reason is dependence of $\gamma$ and $a$ on the crystal direction. It is possible to improve the conductivity by increasing the ratio of lanthanum. When the magnetic alignment is used, it is advantageous to be easily able to control the composition by changing the ratio of the starting powders. Accordingly, it is expected that the electric conductivity can be further improved by controlling the ratio of lanthanum and the $c$-axis orientation simultaneously.

The terminal voltage during fuel-cell performance test is plotted with the power density as a function of the current density in Fig. 10 to evaluate the performances of the single cells. It should be noted that the power density of the cell which consisted of the $c$-axis oriented electrolyte was three times larger compared to that of the cell which consisted of the randomly oriented electrolyte. Herein, the efficiency of the $c$-axis orientation of LSO electrolyte in the cell is reported for the first time. This result demonstrated that the power density can be improved by aligning the $c$-axis that possesses highly conductivity. However, the maximum power density was as low as $\sim 10 \mathrm{~mW} \cdot \mathrm{cm}^{-2}$ at $1000^{\circ} \mathrm{C}$. The reason for the low power density is due to the low performance of electrodes. Since Pt was used as the electrode in this study, the reason for the low output density is considered to be that $\mathrm{Pt}$ was not a highly active material. It is likely that the temperature used to attach Pt on the electrolyte was too high 

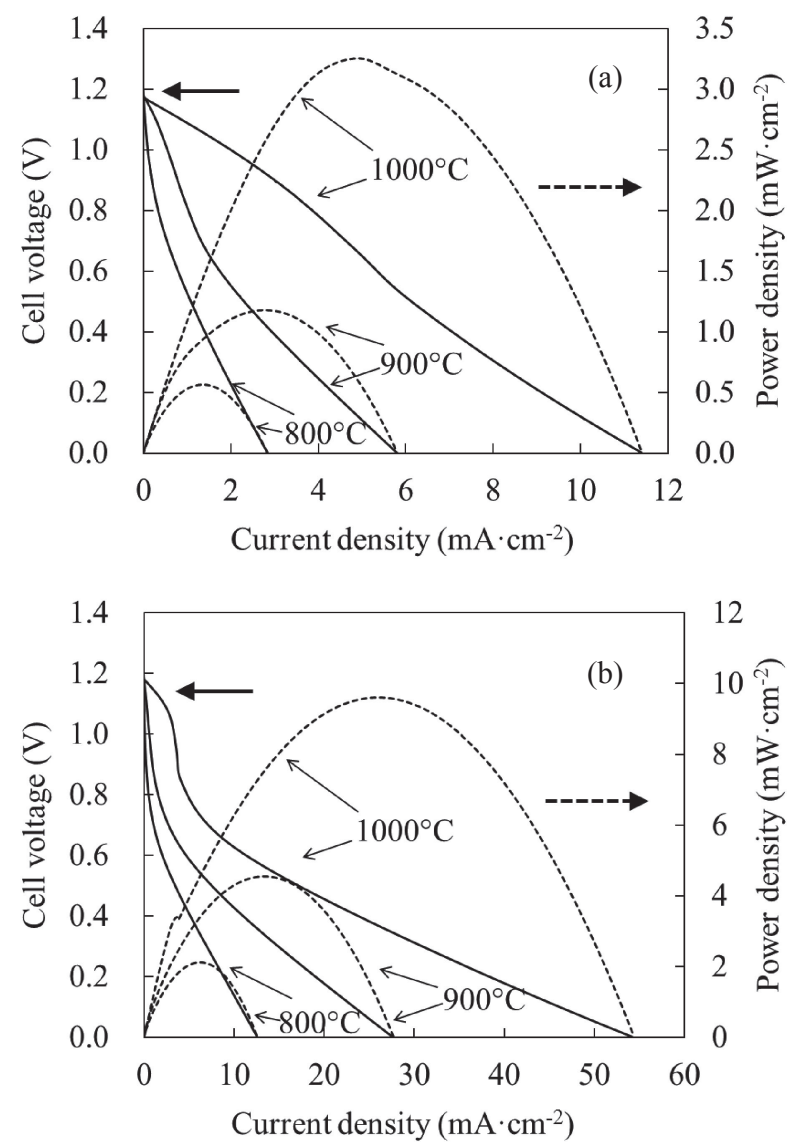

Fig. 10 Cell performance of the LSO prepared by slip casting outside or in a magnetic field followed by sintering at $1600^{\circ} \mathrm{C}$.

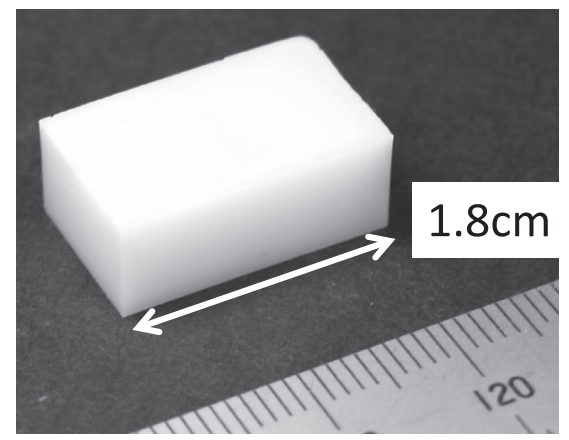

Fig. 11 Photograph of sintered bulk body.

to maintain the electrode's porosity. Conversely, an oxide electrode or a nickel cermet electrode, which is commonly used in a general SOFC, must be used by considering its reactivity with the LSO electrolyte. It has been reported that the $\mathrm{LaCoO}_{3}$ perovskite material is available for use as an air electrode, ${ }^{18)}$ and it has been considered that power generation can be improved based on the selection of the electrode material.

As shown in Fig. 11, it is possible to fabricate a sintered body and to cut a sample from the bulk with the size of its longer dimension equal to $18 \mathrm{~mm}$ after grinding. According to this method, it is possible to produce such a sintered and thick bulk body where its electric conductivity can be measured in the directions not limited to being parallel but also perpendicular to the $c$-axis. In our future work, we will measure the mechanical properties necessary for actual fuel cell applications.

\section{Conclusions}

$C$-axis oriented polycrystal $\mathrm{La}_{9.33}\left(\mathrm{SiO}_{4}\right)_{6} \mathrm{O}_{2}$ bulk ceramics were fabricated by magnetic field-assisted colloidal processing. Regarding the degree of orientation, $77 \%$ of the grains were aligned with tilt angles of less than $20^{\circ}$. The conductivity along the direction of the $c$-axis was higher than those along the other directions. These findings were similar to those reported for single crystals. The cell performance of the LSO electrolyte oriented along the $c$-axis was three times higher than that in the cell with a randomly oriented electrolyte.

\section{Acknowledgement}

This work was partially supported by JSPS KAKENHI grant numbers JP25289264, JP26420690, and JP26420732.

\section{REFERENCES}

1) S. Nakayama, H. Aono and Y. Sadaoka: Chem. Lett. (1995) 431-432.

2) S. Nakayama, T. Kageyama, H. Aono and Y. Sadaoka: J. Mater. Chem. 5 (1995) 1801-1805.

3) S. Nakayama and M. Sakamoto: J. Eur. Ceram. Soc. 18 (1998) 14131418 .

4) K. Kobayashi and C. Nishimura: ECS Trans. 25 (2009) 1785-1790.

5) S. Nakayama, M. Sakamoto, M. Higuchi, K. Kodaira, M. Sato, S. Kakita, T. Suzuki and K. Itoh: J. Eur. Ceram. Soc. 19 (1999) 507-510

6) M. Higuchi, H. Katase, K. Kodaira and S. Nakayama: J. Cryst. Growth 218 (2000) 282-286.

7) M. Higuchi, K. Kodaira and S. Nakayama: J. Cryst. Growth 216 (2000) 317-321.

8) M. Higuchi, Y. Masubuchi, S. Nakayama, S. Kikkawa and K. Kodaira: Solid State Ionics 174 (2004) 73-80.

9) Y. Masubuchi, M. Higuchi, H. Katase, T. Takeda, S. Kikkawa, K. Kodaira and S. Nakayama: Solid State Ionics 166 (2004) 213-217.

10) Y. Masubuchi, M. Higuchi, S. Kikkawa, K. Kodaira and S. Nakayama: Solid State Ionics 175 (2004) 357-360.

11) H. Okudera, A. Yoshiasa, Y. Masubuchi, M. Higuchi and S. Kikkawa: Zeitschrift für Kristallographie - Crystalline Materials 219 (2004) 2731.

12) H. Okudera, A. Yoshiasa, Y. Masubuchi, M. Higuchi and S. Kikkawa: J. Solid State Chem. 177 (2004) 4451-4458.

13) Y. Matsushita, F. Izumi, K. Kobayashi, N. Igawa, H. Kitazawa, Y. Oyama, S. Miyoshi and S. Yamaguchi: Nucl. Instrum. Methods Phys. Res. A 600 (2009) 319-321.

14) S. Nakayama, A. Ikesue, Y. Higuchi, M. Sugawara and M. Sakamoto: J. Eur. Ceram. Soc. 33 (2013) 207-210.

15) K. Fukuda, T. Asaka, S. Hara, M. Oyabu, A. Berghout, E. Bechade, O. Masson, I. Julien and P. Thomas: Chem. Mater. 25 (2013) 2154-2162.

16) S. Nakayama, Y. Higuchi, M. Sugawara, A. Makiya, K. Uematsu and M. Sakamoto: Ceram. Int. 40 (2014) 1221-1224.

17) G. Suarez, N.T.K. Nguyen, N.M. Rendtorff, Y. Sakka and T. Uchikoshi: Ceram. Int. 42 (2016) 19283-19288.

18) S. Mihara, K. Kobayashi, T. Akashi and Y. Sakka: Key Eng. Mater. 616 (2014) 120-128. 